Therefore, we developed a new algorithm for automated and standardised analysis of multiplex FCM data.

Materials and Methods Automation included asinh-transformation of data, cell grouping, population detection and population feature extraction. For grouping of cells, an unbiased unsupervised model based t-mixture approach with Expectation Maximisation (EM)iteration was applied. Populations were detected and identified by meta-clustering of several experiments according to position and extension of cell-clusters in multi-dimensional space and by including a General Procrustes Analysis (GPA) step. For validation, peripheral leukocytes from healthy donors and patients with rheumatoid arthritis (RA) were prepared by hypoosmotic erythrocyte lysis and stained with different sets of lineage-specific antibodies, including CD3, CD4, CD8, CD56, CD19, CD14 and CD15. In parallel, different leukocyte samples were depleted of one of these populations by magnetic beads. Qualitative and quantitative characteristics of major populations were compared with conventional manual analysis.

Results Whole blood leukocytes stained simultaneously with up to 7 markers were correctly distinguished in all major populations including granulocytes (CD15+), T-cells and their subpopulations $(\mathrm{CD} 3+, \mathrm{CD} 4+, \mathrm{CD} 8+)$, monocytes $(\mathrm{CD} 14+)$, B-cells $(\mathrm{CD} 19+)$, and NK-cells (CD56+). The result was comparable to the "gold standard" of manual evaluation by an expert. The new technology is able to detect subclusters and to characterise so far neglected smaller populations based on the new parameters generated. Automated clustering did not require fluorescence compensation of data. Cell-grouping is applicable even for large FCM datasets of at least 10 parameters and more than 1 million events. Comparing the cell-clusters between RA and healthy controls, differences were detectable in several cell (sub-)populations, stable enough to perform correct classification into controls and disease.

Conclusions Our approach reveals first promising results for the analysis of large datasets as generated by multiplex FCM analysis in an automated and time-saving way. Defined clustering algorithms avoid operator-induced bias. In addition, our unsupervised procedure is able to detect unexpected sub-clusters and to characterise so far neglected smaller populations, which may help not only to distinguish normal from disease but also to develop markers for disease activity and therapeutic stratification.

Acknowledgement BTCure IMI grant agreement no. 115142.

\section{A10.5 COMPARATIVE EFFECTIVENESS OF BIOLOGICAL THERAPIES IN RHEUMATOID ARTHRITIS IS INFLUENCED BY RESPONSE MEASURES AND DISEASE ACTIVITY STATE}

\section{doi:10.1136/annrheumdis-2013-203224.5}

\begin{abstract}
1,2Vasco C Romão, ${ }^{2,3}$ Maria José Santos, ${ }^{3} J o s e ́$ Canas da Silva, ${ }^{1,2}$ Joaquim Polido Pereira, ' José Alberto Pereira da Silva, ${ }^{4}$ Cátia Duarte, ${ }^{4}$ José António Pereira da Silva,

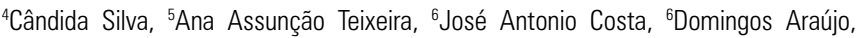
${ }^{7}$ Fernando Pimentel Santos, 7 Jaime Branco, ${ }^{5} J o s e ́$ António Melo Gomes, ${ }^{5}$ Augusto Faustino, 1,2João Eurico Fonseca, 1,2Helena Canhão. 'Rheumatology Department, Lisbon Academic Medical Centre, Portugal; ${ }^{2}$ Rheumatology Research Unit, Instituto de Medicina Molecular, Faculdade de Medicina da Universidade de Lisboa, Lisbon, Portugal; ${ }^{3}$ Rheumatology Department, Hospital Garcia de Orta, Almada, Portugal; ${ }^{4}$ Rheumatology Department, Centro Hospitalar e Universitário de Coimbra, Coimbra, Portugal; 5 Instituto Português de Reumatologia, Lisbon, Portugal; ' ${ }^{6}$ Rheumatology Department, Hospital Conde de Bertiandos, Unidade Local de Saúde do Alto Minho, Ponte de Lima, Portugal; ${ }^{7}$ Rheumatology Department, Hospital de Egas Moniz, Centro Hospitalar Lisboa Ocidental, Lisbon, Portugal
\end{abstract}

Background and Objectives Several biological therapies have become available in the last years in the management of rheumatoid arthritis (RA). Two of the most common drug classes include antitumour necrosis factor (TNF) and anti-interleukin-6 (IL-6) agents, which target central cytokines in the disease pathway. We have previously shown that the proportion of patients achieving remission was higher in the tocilizumab group, an anti-IL-6 agent, compared to anti-TNF therapies, but the magnitude of the effect was associated with the disease activity measure used, namely DAS28, CDAI or SDAI. The aim of this study is to assess whether this difference remains significant in other RA disease activity states.

Materials and Methods We included biologic-naïve RA patients registered in the Rheumatic Diseases Portuguese Register, Reuma.pt, who have started therapy with anti-TNF (adalimumab, infliximab, golimumab) and anti-IL-6 (tocilizumab) monoclonal antibodies after $1^{\text {st }}$ January 2008. Our primary outcome was the proportion of patients in each disease activity state (remission, low, moderate, high) at 6 months, applying DAS28, CDAI and SDAI. Univariate and multivariate logistic regressions were performed to compare the groups.

Results 220 RA patients were enrolled, 180 treated with anti-TNF monoclonal antibodies and 40 treated with tocilizumab. Both groups had similar baseline characteristics but tocilizumab-treated patients had significantly higher SJC, DAS28, SDAI and CDAI as well as shorter disease duration. At 6 months, a significantly higher proportion of patients in the tocilizumab group had reached the DAS28 ( $\mathrm{n}=21$, OR $0.16, \mathrm{p}<0.0001,95 \%$ CI $0.06-0.38)$ and SDAI $(\mathrm{n}=9$, OR $0.29, \mathrm{p}=0.03,95 \% \mathrm{CI} 0.09-0.91)$ remission thresholds, but no significant difference was seen for CDAI $(n=8$, OR 0.41 , $\mathrm{p}=0.12$ ), in the adjusted logistic multivariate model. Moreover, the proportion of patients with moderate $(n=85, O R 3.49, p=0.006$, $95 \%$ CI 1.44-8.43) and high disease activity $(n=30$, OR 6.13, $\mathrm{p}=0.028,95 \% \mathrm{CI} 1.32-30.89$ ) was higher in the anti-TNF group only according to DAS28. No differences were seen in the low disease activity class.

Conclusions Globally, tocilizumab-treated patients had better disease activity outcomes, but the magnitude of the effect was dependent on the disease activity measure used, confirming our previous results and underlining the pronounced reduction of inflammatory markers such as ESR and CRP, translated by lower DAS28 and SDAI, respectively. Furthermore, this effect was also related to the disease activity state considered. This may be explained by the fact that these different indexes distinctly weigh the different components and/or do not classify the same patients in the same disease activity state.

\section{A10.6 DISSECTING DISEASE-SPECIFIC DIFFERENCES IN RA AND OA BY TRANSCRIPTOME ANALYSES OF SYNOVIAL TISSUE, BLOOD AND BONE MARROW MONOCYTES}

doi:10.1136/annrheumdis-2013-203224.6

${ }^{1}$ Biljana Smiljanovic, ${ }^{1}$ Bruno Stuhlmüller, ${ }^{3}$ Wlodzimierz Maslinski, 'Gerd-R Burmester, ${ }^{2}$ Andreas Radbruch, ${ }^{2}$ Andreas Grützkau, 'Thomas Häupl. 'Department of Rheumatology and Clinical Immunology, Charité CCM, Charitéplatz 1, 10117 Berlin, Germany; ${ }^{2}$ German Rheumatism Research Center-Berlin (DRFZ-Berlin), Ein Leibniz-Institut, Charitéplatz 1, 10117 Berlin, Germany; ${ }^{3}$ Department of Pathophysiology and Immunology, Institute of Rheumatology, Warsaw, Poland

Background and Objectives Rheumatoid arthritis (RA) is a chronic inflammatory disease associated with joint destruction. Joint inflammation is characterised by infiltration and activation of various immune cells. To avoid difficulties in sampling synovial tissue and to avoid fluctuation in cellular composition of leukocytes, which accompanies inflammation both in synovial tissue and blood, this study was focused on transcriptome analyses of synovial tissues, blood and bone marrow monocytes. The main aim was to analyse potential of blood monocytes in dissecting inflammation in $\mathrm{RA}$ and in reflecting inflammation that is evident in synovial tissue. Osteoarthritis (OA), which represents a non-inflammatory disease was used as control in this study.

Materials and Methods Synovial tissues from $10 \mathrm{RA}$ and $10 \mathrm{OA}$ patients were used for gene-expression profiling by Affymetrix HGU133A arrays. Blood and bone marrow monocytes, obtained from 